\title{
A prospective observational study of developmental outcomes in survivors of neonatal hypoxic ischaemic encephalopathy in South Africa
}

\author{
D E Ballot, ${ }^{1,2}$ FC Paed (SA), PhD; D Rakotsoane, ${ }^{1}$ FC Paed (SA), Cert Neonatology (SA); P A Cooper, ${ }^{1}$ FC Paed (SA), PhD; \\ T D Ramdin, ${ }^{1,2}$ FC Paed (SA), Cert Neonatology (SA); T Chirwa, ${ }^{3} \mathrm{PhD}$; M S Pepper, ${ }^{2,4} \mathrm{PhD}$ \\ ${ }^{1}$ Department of Paediatrics and Child Health, School of Clinical Medicine, Faculty of Health Sciences, University of the Witwatersrand, \\ Johannesburg, South Africa \\ ${ }^{2}$ Neonatal Encephalopathy Suspected Hypoxic Ischaemic Encephalopathy (NESHIE) collaboration, South Africa \\ ${ }^{3}$ Division of Epidemiology and Biostatistics, School of Public Health, Faculty of Health Sciences, University of the Witwatersrand, Johannesburg, \\ South Africa \\ ${ }^{4}$ SAMRC Extramural Unit for Stem Cell Research and Therapy, Department of Immunology, Faculty of Health Sciences, University of Pretoria, \\ South Africa
}

Corresponding author: D E Ballot (daynia.ballot@wits.ac.za)

\begin{abstract}
Background. Neonatal hypoxic ischaemic encephalopathy (NHIE) is an important cause of long-term handicap in survivors. There is limited information on the burden of handicap from NHIE in sub-Saharan Africa.

Objectives. To determine the developmental outcomes in survivors of NHIE in South Africa (SA).

Methods. In this prospective observational study, the developmental outcomes in 84 infants who had survived hypoxic ischaemic encephalopathy (the NHIE group) were compared with those in 64 unaffected infants (the control group). The Bayley Scales of Infant Development version III were used for assessment of developmental outcomes.

Results. Significant differences were found between the developmental outcomes of the two groups, with a significantly lower composite language score and higher proportions with language, motor and cognitive developmental delays in the NHIE group than in the control group. Cerebral palsy (CP) was present in 13 of the infants with NHIE $(15.5 \%)$ and none in the control group $(p<0.001)$. CP was associated with developmental delay, and also with the severity of NHIE. Therapeutic hypothermia (TH) was administered in $58.3 \%$ of the study group, but although it was associated with lower rates of $\mathrm{CP}$ and developmental delay than in the group without $\mathrm{TH}$, the only significant difference was for delay on the language subscale.

Conclusions. Survivors of NHIE in SA are at risk of poor developmental outcomes.

S Afr Med J 2020;110(4):308-312. https://doi.org/10.7196/SAMJ.2020.v110i4.14311
\end{abstract}

Intrapartum asphyxia and consequent neonatal hypoxic ischaemic encephalopathy (NHIE) is a problem in low- and middle-income countries (LMICs). It has been suggested that $<10 \%$ of cases of cerebral palsy $(\mathrm{CP})$ in high-income countries are caused by intrapartum asphyxia. ${ }^{[1]}$ However, intrapartum asphyxia may be the cause of up to $42 \%$ of CP cases in LMICs. ${ }^{[2]}$ The strong relationship between $\mathrm{CP}$ and intrapartum asphyxia in LMICs may be due to under-resourcing and lack of access to appropriate health facilities. The incidence of NHIE varies considerably, with the greatest burden occurring in sub-Saharan Africa (14.9 per 1000 live births) and much lower rates (1.5 per 1000 live births) in high-income countries. ${ }^{[3]}$ Results from previous studies conducted in tertiary referral centres in South Africa (SA) have demonstrated NHIE rates of $2.3-13.3$ per 1000 live births. ${ }^{[4-6]}$ The rate of NHIE is likely to be considerably higher in rural areas, where there are relatively high levels of home births and health facilities are often under-resourced.

Therapeutic hypothermia (TH) improves the outcomes associated with NHIE. A Cochrane review of 11 studies demonstrated that $\mathrm{TH}$ reduces both death and major neurodevelopmental disability. ${ }^{[7]}$ Although there is some controversy regarding the safety and benefits of TH in LMICs, ${ }^{[8]}$ it has been provided in the public sector in SA for several years.
There is a lack of information on developmental outcomes in survivors of NHIE in SA. A retrospective review conducted in Johannesburg reported that $79.6 \%$ of survivors of NHIE were neurologically normal on follow-up, with a CP incidence of $13.2 \%{ }^{[4]}$ However, no formal neurodevelopmental assessment was performed, and information about developmental outcomes was available for fewer than one-third of the neonates. Accurate information on developmental outcomes and rates of disability in survivors of NHIE is needed to enable planning of further research and to make judgements on the merits of health interventions such as TH.

\section{Objectives}

To compare developmental outcomes in survivors of NHIE with those in healthy children born at full term in a hospital in SA.

\section{Methods}

A prospective, observational study was conducted at a tertiary referral hospital in Johannesburg. Mothers of infants who had survived NHIE and had been discharged from the hospital's neonatal unit between June 2013 and December 2016 were invited to participate in the follow-up study. Those who presented at the follow-up clinic and provided consent to participate were enrolled. 
NHIE was diagnosed in neonates with evidence of encephalopathy and perinatal asphyxia, as listed below under eligibility criteria for cooling. The stage of NHIE (grades 1 - 3) was assessed by the attending paediatric staff, as described previously. ${ }^{[9]}$ Infants who were not assigned an NHIE grade at birth were excluded from the study.

Whole-body cooling was available on a limited basis during the study period. In each case, the attending paediatrician's decision to initiate $\mathrm{TH}$ was based on the unit protocol and the availability of a cooling machine. The unit protocol, which was modified from that used in the TOBY study, has been described elsewhere. ${ }^{[10,11]}$ Eligibility criteria for cooling were birthweight $>2000$ g, gestational age $>36$ weeks, a history suggestive of perinatal asphyxia, and at least one of the following conditions: the need for ongoing resuscitation at birth for $>10$ minutes, evidence of metabolic acidosis (base excess $\leq 16 \mathrm{mmol} / \mathrm{L}$ ) on a blood-gas test performed within 1 hour of birth, a 10-minute Apgar score $<5$, and clinical evidence of encephalopathy. Neither amplitude-integrated electroencephalography (aEEG) nor magnetic resonance imaging (MRI) was available during the study period. Cranial ultrasonography was available at most times, but its availability was not a factor that was considered when determining eligibility for inclusion in the study. When used, TH was initiated within 6 hours of birth, in a high-care (level 2) ward. A servocontrolled cooling mattress was used, and the target rectal temperature was $32-34^{\circ} \mathrm{C}$, with gradual rewarming after 72 hours. Neonates with asphyxiation were not routinely provided with mechanical ventilation, according to the unit's neonatal intensive care admission policy, so neonates with severe asphyxia who were apnoeic and required ventilation were not considered suitable candidates for $\mathrm{TH}$.

Infants were seen at a dedicated follow-up clinic every 3 months. Neurodevelopmental assessments were performed by either an appropriately trained physiotherapist or a paediatrician, using the Bayley Scales of Infant Development version III (BSID-III) at 9 12 months of age, and then again at $15-18$ months. The assessor was not aware of the group classification (NHIE or control). The different observers' assessments had a Cronbach's alpha intra-class correlation of 0.89 . The initial BSID-III assessment was performed in anticipation of infants failing to attend follow-up visits after the age of 12 months. The most recent BSID-III assessment for each participant was used in the data analysis.

$\mathrm{CP}$ was defined as a permanent, non-progressive disorder in the development of movement and posture, which in this study was attributed to neonatal NHIE. ${ }^{[12]}$

The study co-ordinator sent parents of participants text messages to remind them of appointments, to achieve a good rate of followup. The parents were reimbursed for their transport costs at each visit that they attended. The study co-ordinator contacted defaulters by telephone and rescheduled the appointments. If a BSID-III assessment was missed, the assessment was performed at the next follow-up visit that the infant attended. Infants who were noted to have developmental problems were referred to the appropriate allied medical services for treatment.

A group of full-term infants born at the same hospital who were not admitted at birth has been described previously. ${ }^{[13]}$ The infants in the NHIE study group were assessed at a younger mean age than the overall mean in the previously described control group, so a subgroup of 64 healthy, full-term infants who had been tested at the same age as the NHIE study group was used as the control group in this study.

\section{Ethical considerations}

The study was approved by the Human Research Ethics Committee of the University of the Witwatersrand (ref. no. M120623). Written informed consent for participation was obtained from each parent prior to the infant's enrolment in the study.

\section{Data collection}

Demographics, labour room details, clinical characteristics and developmental assessment results were collected for each participant. The data were entered into a computerised database hosted by the University of the Witwatersrand using Research Electronic Data Capture (REDCap) (https://www.project-redcap.org/).[14] Data will be made available on reasonable request to the corresponding author (DEB).

\section{Sample size}

The BSID-III normative population has a mean score of 100 with a standard deviation (SD) of 15 for each subscale. On the basis of the assumption that the mean score of the study population was 89 , with an SD of 15, a minimum sample size of 30 in each group would be required to detect a statistically significant difference between the means, with $\alpha=0.05$ and $\beta=0.80$. Previous follow-up studies in the same population have shown a $25 \%$ default rate, so the required group size was adjusted to 38 participants.

\section{Statistical analysis}

Data were analysed using SPSS version 24 (IBM Corp., USA). Continuous data that were normally distributed were summarised by means and $95 \%$ confidence intervals (CIs), whereas categorical data were summarised by frequencies and percentages. The BSID-III assesses development in the cognitive, language and motor domains. We compared the composite BSID-III scores for each domain between the control and study groups using independent $t$-tests and $\chi^{2}$ tests, as appropriate. A mean composite score $<85$ was defined as 'at risk' and a mean composite score $<70$ as 'delayed. ${ }^{\text {[13] }}$ The number of infants who were at risk or delayed, and the number with $\mathrm{CP}$, were compared between the two groups. Results with $p$-values $<0.05$ were considered statistically significant.

\section{Results}

Among 99 infants who attended at least one follow-up clinic visit, 87 had at least one BSID-III assessment, but 3 did not have a grade of NHIE assigned at birth and were excluded. The final sample therefore comprised 84 infants who had at least one BSID-III assessment, corresponding to a follow-up rate of $84.8 \%$. The BSIDIII assessments were performed at a mean age of 14.31 months (95\% CI 13.27 - 15.34). The mean gestational age of the infants who survived NHIE was 38.98 weeks (95\% CI 38.45 - 39.51), while that of the control group was 39.52 weeks (95\% CI 39.12 - 39.91). The mean birthweight of the NHIE group was $3090 \mathrm{~g}$ (95\% CI 2950 $3230)$ and that of the control group was $2740 \mathrm{~g}$ (95\% CI 2640 $2890 \mathrm{~g})$. The majority of infants were male (60/84; 71.4\%). NHIE grade 1 was present in 14 of the infants (16.7\%), with grade 2 in 56 (66.7\%) and grade 3 in 14 (16.7\%). TH was administered in 49 of the infants (58.3\%). Additional data from the study population are shown in Table 1.

The mean composite scores for the BSID-III cognitive, language and motor subscales for NHIE survivors and controls are shown in Table 2 . The only significant difference between the groups was for the language subscale, on which the control group had a higher score than the study group $(p=0.001)$.

The numbers of participants classified as at risk or delayed were significantly greater in the NHIE group than in the control group for each subscale, with the exception of the numbers at risk on the 
cognitive subscale (Table 3). None of the participants in the control group were classified as delayed.

The number of participants in the NHIE group who had CP ( $n=13$; $15.5 \%)$ was significantly higher than the number in the control group $(n=0)(p<0.001)$. There was no information available on the types of CP. Among the 13 infants with CP, 12 were classified as delayed on the BSID-III motor subscale, and 1 as at risk. Only 1 infant with motor delay did not have evidence of CP. Eight of the infants with $\mathrm{CP}$ had global delay (classified as delay in the cognitive, motor and language domains), and 2 of them were also blind. None of the infants without CP had global delay. The grade of NHIE was not significantly associated with the incidence of CP. One of the 14 infants with NHIE grade 1 (7.1\%) had CP, compared with 9 of the 56 infants with NHIE

\begin{tabular}{ll} 
Table 1. Baseline characteristics of 84 infants who survived \\
neonatal hypoxic ischaemic encephalopathy \\
\hline Variable & $\boldsymbol{n}(\%)$ \\
\hline Born in the study hospital & $69(82.1)$ \\
Primipara & $26(31.0)$ \\
Maternal hypertension & $4(4.8)$ \\
Maternal HIV & $18(21.4)$ \\
Teenage mother & $2(2.4)$ \\
Mode of delivery & \\
Normal vaginal & $39(46.4)$ \\
Assisted vaginal & $6(7.1)$ \\
Vaginal breech & $6(7.1)$ \\
Caesarean section & $43(51.2)$ \\
5-minute Apgar score $<6$ & $51(60.7)$ \\
Resuscitation at birth & $61(73.5)$ \\
Early-onset sepsis & $1(1.2)$ \\
Abnormal cranial ultrasound scan & $4(4.8)$ \\
Meconium aspiration syndrome & $5(6.0)$ \\
Nasal CPAP & $2(2.4)$ \\
Mechanical ventilation & $1(1.2)$ \\
Late-onset sepsis & $5(6)$ \\
CPAP = continuous positive airway pressure ventilation. & \\
\end{tabular}

grade $2(16.1 \%)$ and 3 of the 14 infants with NHIE grade $3(21.4 \%)$ ( $p=0.566, \chi^{2}$ analysis).

The numbers of individuals in the NHIE group who had CP or delay on the BSID-III subscales were analysed according to whether or not TH had been used (Table 4). There were fewer infants with $\mathrm{CP}$ or delay in the group who had received TH compared with those who had not; however, the only significant difference occurred with the language subscale $(p=0.04)$.

\section{Discussion}

The results of our study in Johannesburg have provided important information about developmental outcomes in infants who survive NHIE. Rates of CP and developmental delays determined by the cognitive, motor and language subscales of the BSID-III were all significantly higher in survivors of NHIE than in a control group. The rate of CP that we found in the NHIE survivors was slightly higher than that previously reported at the same location, ${ }^{[4]}$ and much higher than the rate previously found to be associated with preterm birth. ${ }^{[15,16]}$ These results suggest that perinatal asphyxia is the most important cause of CP in sub-Saharan Africa. Measures to prevent perinatal asphyxia and to treat neonates who sustain NHIE should therefore be prioritised.

We observed increasing rates of $\mathrm{CP}$ as the grade of NHIE increased, but this relationship was not statistically significant, possibly because of the limited numbers in the study group. The overall rate of CP in our NHIE group (15.5\%) was similar to a previous report indicating that up to one in every six neonates with moderate-to-severe NHIE will develop CP. ${ }^{[17]}$

The burden of handicap in survivors of NHIE is profound. In the present study, $>60 \%$ of children with CP had global developmental problems, with associated cognitive and language handicap, and in some cases blindness. It is very unlikely that these affected individuals will ever be able to live independently, and they will almost certainly require ongoing lifelong care. Perinatal asphyxia not only results in neurological handicap in survivors but is the most common cause of early death in neonates with birthweight $>1000 \mathrm{~g},{ }^{[18]}$ and is a factor that contributes to the ever-increasing burden of medicolegal litigation. It is therefore important to prioritise interventions to

Table 2. Mean composite BSID-III subscale scores for 84 infants with neonatal hypoxic ischaemic encephalopathy (NHIE group) and 64 unaffected infants (control group)

\begin{tabular}{|c|c|c|c|c|c|}
\hline \multirow[b]{2}{*}{ Variable } & \multicolumn{2}{|c|}{ NHIE group } & \multicolumn{2}{|c|}{ Control group } & \multirow[b]{2}{*}{$p$-value ${ }^{*}$} \\
\hline & Mean & $95 \% \mathrm{CI}$ & Mean & $95 \% \mathrm{CI}$ & \\
\hline Age (months) & 14.3 & $13.3-15.3$ & 13.6 & $12.6-14.6$ & 0.140 \\
\hline Cognitive score & 93.4 & $88.8-97.9$ & 92.5 & $89.5-95.5$ & 0.840 \\
\hline Language score & 92.1 & $87.9-96.3$ & 99.9 & $97.2-102.6$ & 0.001 \\
\hline Motor score & 92.6 & $86.9-98.2$ & 96.6 & $94.1-99.1$ & 0.190 \\
\hline
\end{tabular}

Table 3. Classifications of 'at-risk' or 'delayed' status according to BSID-III subscale scores of 84 infants with neonatal hypoxic ischaemic encephalopathy (NHIE group) and 64 unaffected infants (control group)

\begin{tabular}{|c|c|c|c|c|c|c|}
\hline \multirow[b]{2}{*}{ Subscale } & \multicolumn{2}{|c|}{ At-risk status (score $<85$ ) } & \multirow[b]{2}{*}{$p$-value ${ }^{*}$} & \multicolumn{2}{|c|}{ Delayed status $($ score $<70)$} & \multirow[b]{2}{*}{$p$-value ${ }^{\star}$} \\
\hline & NHIE group, $n(\%)$ & Control group, $n(\%)$ & & NHIE group, $n(\%)$ & Control group, $n(\%)$ & \\
\hline Cognitive & $18(21.4)$ & $14(22.2)$ & 0.94 & $12(14.3)$ & 0 & $<0.001$ \\
\hline Language & $23(27.4)$ & $6(9.3)$ & 0.006 & $8(9.5)$ & 0 & 0.01 \\
\hline Motor & $22(26.2)$ & $5(7.8)$ & 0.005 & $13(15.5)$ & 0 & $<0.001$ \\
\hline
\end{tabular}


Table 4. TH and handicap among survivors of neonatal hypoxic ischaemic encephalopathy

\begin{tabular}{|c|c|c|c|}
\hline Variable & TH $(N=49), n(\%)$ & No TH $(N=35), n(\%)$ & $p$-value ${ }^{*}$ \\
\hline Cerebral palsy & $5(10.2)$ & $8(22.9)$ & 0.11 \\
\hline Motor delay & $5(10.2)$ & $8(22.9)$ & 0.11 \\
\hline Language delay & $2(4.1)$ & $6(17.1)$ & 0.04 \\
\hline Cognitive delay & $5(10.2)$ & $7(20.0)$ & 0.21 \\
\hline
\end{tabular}

reduce the incidence of intrapartum hypoxia. Previous results have highlighted that a significant number of cases of perinatal asphyxia in resource-limited settings are preventable. ${ }^{[19]}$ Issues including inadequate training and insufficient numbers of healthcare workers, high patient loads, inadequate ambulance services and lack of operating theatres result in delays in the transport of women in labour to proper health facilities, and in decisions to conduct caesarean section deliveries. ${ }^{[2]}$ The provision of sufficient resources is therefore necessary for the appropriate care of mothers and babies, to prevent neonatal mortality and morbidity. ${ }^{[2]}$ From a paediatric perspective, ensuring prompt and effective neonatal resuscitation at all levels of care would greatly improve outcomes related to intrapartum hypoxia. ${ }^{[20]}$ Evidence indicates that basic neonatal resuscitation is beneficial, and is possible even in resource-limited settings. ${ }^{[20]}$

There are a number of challenges to providing $\mathrm{TH}$ in a resourcelimited setting, including the arrival of neonates at the cooling centre after the 6-hour postpartum window, shortages of staff and equipment, and limited availability of ventilation facilities. However, results from a study conducted in Morocco suggest that TH is both feasible and safe in LMICs. ${ }^{[21]} \mathrm{TH}$ has been provided in the public sector in SA for several years, but its benefits in resource-limited settings are still being debated. In a systematic review and meta-analysis of the benefits of TH in LMICs, no reduction in neonatal morbidity was demonstrated, and it was concluded that the safety and efficacy of cooling in LMIC settings must be confirmed before it can be provided routinely ${ }^{[8]}$ In contrast, a meta-analysis of three studies of low-technology cooling methods (gel packs) in intensive care settings showed reductions in both mortality and neurodevelopmental handicap in neonates who sustained intrapartum hypoxia. ${ }^{[22]}$ However, frequent measurement and adjustment of the temperature is required with these methods, so these findings are not generalisable to situations with severe resource limitations where there are shortages of nursing staff and equipment.

The present study was not primarily designed to determine the effects of TH on outcomes associated with NHIE, and was therefore not sufficiently powered for this purpose. However, we did observe a significant reduction in the occurrence of language disabilities, as well as a trend towards lower rates of $\mathrm{CP}$ and cognitive delays, in survivors of NHIE who had received TH. No increase in the rate of developmental delay or disability was observed in the $\mathrm{TH}$ group compared with the non- $\mathrm{TH}$ group, suggesting that $\mathrm{TH}$ is a safe procedure. A publication from Tygerberg Hospital in Cape Town that reviewed all neonates with NHIE treated with TH found that $18 \%$ of survivors had severe neurodevelopmental handicap at 1 year of age. ${ }^{[23]}$ The Tygerberg study did not have a control group, but the reported rate of handicap is similar to that in the NHIE survivors in the present study, so it is unclear whether TH is beneficial in a resource-limited setting. To determine the precise benefits of $\mathrm{TH}$ in resource-limited settings, further research needs to be carried out, such as the 'hypothermia for encephalopathy in low- and middle-income countries' (HELIX) trial, a randomised controlled trial of TH v. standard care that is currently underway in Bangladesh, India and Sri Lanka. ${ }^{[2]}$

\section{Study limitations}

This study was conducted at a single centre, and the numbers in the sample are small. Much larger study populations are required to fully evaluate the consequences of neonatal NHIE, particularly CP. The small scale of the current study did not enable in-depth analysis of the effects of TH on different grades of NHIE. A multicentre collaborative study that is adequately powered to evaluate these outcomes is recommended. The diagnosis and grading of NHIE in the current study was not standardised, and aEEG and MRI investigations were not available. Although a reasonable rate of follow-up was achieved, it is possible that those who defaulted could have been handicapped, which is a potential source of bias in the results.

\section{Generalisability}

This study was conducted in a relatively well-resourced setting and the findings may not be generalisable to under-served settings in the rest of sub-Saharan Africa, where the rates of handicap and $\mathrm{CP}$ are likely to be far higher.

\section{Conclusions}

Perinatal asphyxia and consequent NHIE is an important cause of developmental delays, including CP, in infants in sub-Saharan Africa. Measures to prevent and treat this condition should be prioritised. Further research needs to be conducted to establish whether TH reduces neurodevelopmental handicap and mortality in resourcelimited settings. Other research questions include the selection of appropriate candidates for $\mathrm{TH}$, adjuvant therapies appropriate for low-resourced settings, whether or not $\mathrm{TH}$ should be provided without mechanical ventilation, and the cost benefit of TH in lowresourced settings.

\section{Declaration. None.}

Acknowledgements. The authors gratefully acknowledge the assistance of Mr L Rapola and Mr M Reineke in data capture, Ms Hanrahan and Dr M Ally in co-ordinating the clinic, and Dr N Brown and Dr L Madzudzo in data collection.

Author contributions. DEB conceptualised the study, obtained the study grant, collected and analysed the data, and wrote the various drafts and final submission of the manuscript. DR assisted with the study design, data collection and critical review of the drafts and final submission. PAC assisted with the study design and critical review of the drafts and final submission. TR assisted with data collection and critical review of the drafts and final submission. TC assisted with the study design, data analysis and critical review of the drafts and final submission. MP reviewed the study design and critically reviewed the drafts and final submission.

Funding. This study was funded by a self-initiated research grant from the South African Medical Research Council.

Conflicts of interest. None. 
1. Jacobsson B, Hagberg G. Antenatal risk factors for cerebral palsy. Best Pract Res Clin Obstet Gynaecol 2004;18(3):425-436. https://doi.org/10.1016/j.bpobgyn.2004.02.011

2. Cooper PA. Cerebral palsy in SA: Perinatal factors and later neurological handicap in infants born at or near term. S Afr J Child Health 2015;9(4):1. https://doi.org/10.7196/sajch.2015.v9i4.1064

3. Lee AC, Kozuki N, Blencowe $\mathrm{H}$, et al. Intrapartum-related neonatal encephalopathy incidence and impairment at regional and global levels for 2010 with trends from 1990. Pediatr Res 2013;74(Suppl 1):50-72. https://doi.org/10.1038/pr.2013.206

4. Padayachee N, Ballot DE. Outcomes of neonates with perinatal asphyxia at a tertiary academic hospital in Johannesburg, South Africa. S Afr J Child Health 2013;7(3):89-94. https://doi.org/10.7196/ SAJCH.574 5. Horn AR, Swingler GH, Myer L, et al. Early clinical signs in neonates with hypoxic ischemic
encephalopathy predict an abnormal amplitude-integrated electroencephalogram at age 6 hours. BMC Pediatr 2013;13:52. https://doi.org/10.1186/1471-2431-13-52

6. Bruckmann EK, Velaphi S. Intrapartum asphyxia and hypoxic ischaemic encephalopathy in a public hospital: Incidence and predictors of poor outcome. S Afr Med J 2015;105(4):298-303. https://doi. org/10.7196/SAMI.9140

7. Jacobs SE, Berg M, Hunt R, Tarnow-Mordi WO, Inder TE, Davis PG. Cooling for newborns with hypoxic ischaemic encephalopathy. Cochrane Database Syst Rev 2013, Issue 1. Art. No.: CD003311. https://doi.org/10.1002/14651858.CD003311.pub3

8. Pauliah SS, Shankaran S, Wade A, Cady EB, Thayyil S. Therapeutic hypothermia for neonatal encephalopathy in low- and middle-income countries: A systematic review and meta-analysis. PloS One 2013;8(3):e58834. https://doi.org/10.1371/journal.pone.0058834

9. Sarnat HB, Sarnat MS. Neonatal encephalopathy following fetal distress: A clinical and electroencephalographic study. Arch Neurol 1976;33(10):696-705. https://doi.org/10.1001/ archneur. 1976.00500100030012

10. Sebetseba KN, Ramdin T, Ballot D. The use of therapeutic hypothermia in neonates with perinatal asphyxia at Charlotte Maxeke Johannesburg Academic Hospital: A retrospective review. Ther asphyxia at Charlotte Maxeke Johannesburg Academic Hospital: A retrospective review. Ther
Hypothermia Temp Manag 2017 (epub 28 November 2017). https://doi.org/10.1089/ther.2017.0040

11. Azzopardi D, Brocklehurst P, Edwards D, et al.; The TOBY Study Group. The TOBY Study. Whole bod hypothermia for the treatment of perinatal asphyxial encephalopathy: A randomised controlled trial. BMC Pediatr 2008;8:17. https://doi.org/10.1186/1471-2431-8-17

12. Richards CL, Malouin F. Cerebral palsy: Definition, assessment and rehabilitation. Handb Clin Neurol 2013;111:183-195. https://doi.org/10.1016/B978-0-444-52891-9.00018-X

13. Ballot DE, Ramdin T, Rakotsoane D, et al. Use of the Bayley Scales of Infant and Toddler Development, third edition, to assess developmental outcome in infants and young children in an urban setting in South Africa. Int Sch Res Notices 2017;2017:1631760. https://doi.org/10.1155/2017/1631760
14. Harris PA, Taylor R, Thielke R, Payne J, Gonzalez N, Conde JG. Research electronic data capture (REDCap) - a metadata-driven methodology and workflow process for providing translational (REDCap) - a metadata-driven methodology and workflow process for providing translational
research informatics support. J Biomed Inform 2009;42(2):377-381. https://doi.org/10.1016/j. research informa

15. Ballot DE, Potterton J, Chirwa T, Hilburn N, Cooper PA. Developmental outcome of very low birth weight infants in a developing country. BMC Pediatr 2012;12:11. https://doi.org/10.1186/1471-2431weig $12-1$

16. Ballot DE, Ramdin T, Rakotsoane D, et al. Assessment of developmental outcome in very low birth weight infants in southern Africa using the Bayley Scales of Infant Development (III). BMJ Paediat Open 2017;1(1):e000091. https://doi.org/10.1136/bmjpo-2017-000091

17. Ahearne CE, Boylan GB, Murray DM. Short and long term prognosis in perinatal asphyxia: An update. World J Clin Pediatr 2016;5(1):67-74. https://doi.org/10.5409/wjcp.v5.i1.67

18. Pattinson P, Rhoda N, PPIP Group. Saving Babies 2012 - 2013: Ninth Report on Perinatal Care in South Africa. Pretoria: Tshepesa Press, 2014. https://www.ppip.co.za/wp-content/uploads/ SavingBabies-2012-2013.pdf (accessed 23 February 2019).

19. Gregersen NE, Ballot DE, Guidozzi F, Cooper PA. Birth asphyxia - presenting the case for a 'stitch in time. S Afr Med J 1999;89(3):326-332.

20. Wall SN, Lee AC, Carlo W, et al. Reducing intrapartum-related neonatal deaths in low- and middleincome countries - what works? Semin Perinatol 2010;34(6):395-407. https://doi.org/10.1053/j. semperi.2010.09.009

21. Maoulainine FMR, Elbaz M, Elfaiq S, et al. Therapeutic hypothermia in asphyxiated neonates: Experience from neonatal intensive care unit of University Hospital of Marrakech. Int J Pediat 2017;2017:3674140. https://doi.org/10.1155/2017/3674140

22. Rossouw G, Irlam J, Horn AR. Therapeutic hypothermia for hypoxic ischaemic encephalopathy using low-technology methods: A systematic review and meta-analysis. Acta Paediatr 2015;104(12):12171228. https:///oi.org/10.1111/apa.12830

23. Kali GT, Martinez-Biarge M, van Zyl J, Smith J, Rutherford M. Therapeutic hypothermia for neonatal hypoxic-ischaemic encephalopathy had favourable outcomes at a referral hospital in a middle-income country. Acta Paediatr 2016;105(7):806-815. https://doi.org/10.1111/apa.13392

24. Thayyil S, Oliveira V, Lally PJ, et al. Hypothermia for encephalopathy in low and middle-income countries (HELIX): Study protocol for a randomised controlled trial. Trials 2017;18(1):432. https:/ doi.org/10.1186/s13063-017-2165-3

Accepted 25 September 2019. 